\title{
Organic Diselenides: Versatile Reagents, Precursors, and Intriguing Biologically Active Compounds
}

\author{
Claudio Santia ${ }^{\star}$, Cristina Tomassini ${ }^{a}$, and Luca Sancineto ${ }^{\mathrm{b}}$
}

\begin{abstract}
In this account, we describe how some organic diselenides were successfully used in the past as reagents for asymmetric stereoselective synthesis and more recently as precursors of catalysts and reagents applied in new green protocols. A biomimetic approach offered the possibility to perform oxidative reactions using hydrogen peroxide as oxidant and water as medium affording the desired products in excellent yields under mild conditions. The umpolung of the selenium atom gave novel nucleophilic reagents having a strongly accelerated reaction rate in on water conditions. Finally, the use of diselenides to exploit specific biological activities is described here as seminal examples of a promising field of research currently under investigation in our group.
\end{abstract}

Keywords: Catalysis $\cdot$ Electrophile $\cdot$ Nucleophile $\cdot$ Redox $\cdot$ Selenium

\section{Introduction}

During the last decades organoselenium derivatives have become attractive compounds for several research groups interested in chemo-, regio- and stereoselective synthesis, catalysis, green chemistry as well as in many biological activities that were recently proved for some selenium-containing molecules. ${ }^{[1]}$ The introduction of an organoselenium moiety into an organic substrate can be achieved using electrophilic, nucleophilic, or radical selenium species that are readily accessible starting from the corresponding diselenides by oxidation, reduction or homolytic cleavage of the Se-Se bond, respectively. Diselenides, compared to the corresponding reactive derivatives, are considerably easier to handle and are normally stable under atmospheric conditions. This account will take in consideration some aspects of our research that in the last 20 years have dealt with the use of diselenides in asymmetric synthesis, catalysis, reaction in on water conditions and the development of new biologically active selenium compounds. Because many of these results have already been collected in review articles and book chapters, ${ }^{[2]}$ this account will be more focused on some recent developments.

\section{Enantiomerically Pure Diselenides in Asymmetric Synthesis}

Since the early 1990 s the optimization of a series of efficient procedures to prepare enantiomerically pure diselenides allowed their use in asymmetric synthesis as catalysts in metal-mediated conversion as well as precursors of electrophilic reagents successfully applied in various stereoselective selenoaddition reactions. In both cases the presence of a neighbouring heteroatom to the selenium proved to be mandatory for the selectivities. In the first case, the heteroatom cooperates with selenium on metal chelation while in the second it establishes a non-bonding interaction with the electrophilic centre affording more rigid transition states and bringing the chiral moiety near to the reaction centre during the nucleophilic attack of the double bond.
Some examples of diselenides that were successfully used in asymmetric synthesis are reported in Fig. 1.

Diselenides $\mathbf{1}$ and $\mathbf{2}$ were used as catalysts for the diethyl zinc addition to benzaldehydes affording the corresponding alcohol in $96 \%$ and $97 \%$ ee, respectively. This evidenced a positive effect of both the second chiral centre (2) and of a bulky group in suitable position to force the heteroatom close to selenium facilitating the coordination of the zinc $(\mathbf{1}) .^{[3,4]}$

Concerning the electrophilic addition to $\mathrm{C}=\mathrm{C}$, the nature of the heteroatom as well as the anion of the positively charged selenium was demonstrated to be crucial in term of reactivity and selectivity. ${ }^{[2,5]}$ Sulfur-containing diselenides were proven to be the most efficient, affording many intra- and intermolecular addition reactions generally in good yield and good stereoselectivities in milder reaction conditions compared to nitrogen- or oxygencontaining derivatives. Among the several stereoselective conversions reported using electrophilic reagents generated by $\mathbf{4}$ and $\mathbf{5}$ (Fig. 1), particularly interesting was the first example of azido selenenylation

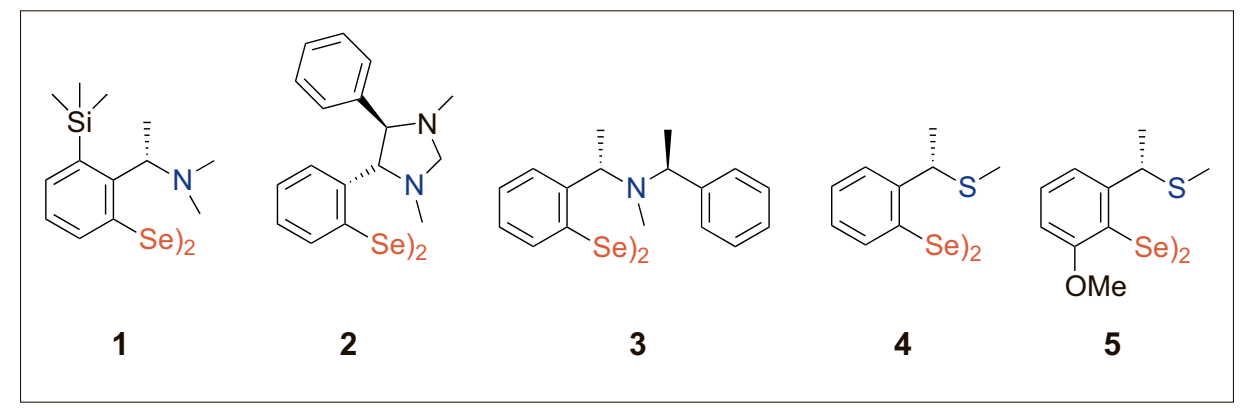

Fig. 1. Selected examples of enantiomerically pure diselenides. 


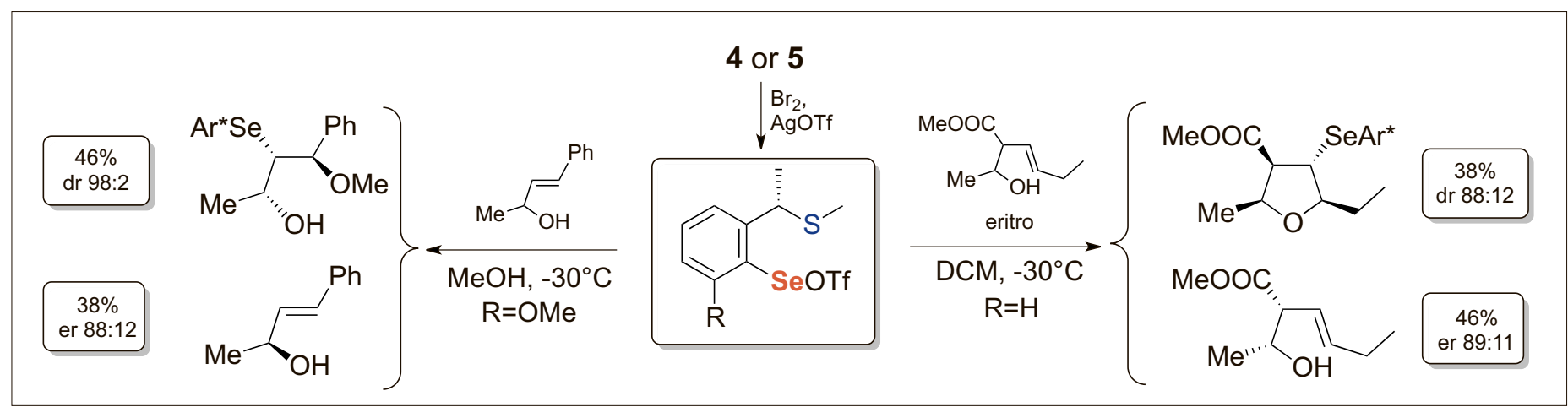

Scheme 1. Kinetic resolutions using enantiomerically pure diselenides.

Scheme 2. Chiral diselenides in asymmetric catalysis.

that allowed the preparation of a series of optically enriched nitrogen-containing derivatives.[6] To the best of our knowledge we also reported the first example of non-enzymatic kinetic resolution of allylic alcohols through an electrophilic methoxyselenenylation reaction ${ }^{[7]}$ and, more recently, the kinetic resolution of 2-cabomethoxy-3-alkenols exploiting a cyclofunctionalization with the formation of functionalized tetrahydrofurans. ${ }^{[8]}$ In both cases half equivalent of electrophilic reagent activates the stereoselective interor intramolecular addition to the double bond leaving unreacted half equivalent of enantiomerically enriched starting material. The yields are generally good and the enantiomeric excess ranges from $70 \%$ up to $95 \%$ (Scheme 1)

An interesting two-step, one-pot selenenylation-deselenenylation reaction was performed using ammonium persulfate as oxidant for both steps: i) it generates the electrophilic species starting from a diselenide and ii) it activates the selenide intermediate toward the elimination or the substitution. Using this protocol a catalytic amount of diselenide $\mathbf{3}$ was employed to transform $\beta, \gamma$-unsaturated ester 6 into the corresponding allylic ether 7 in $50 \%$ yield and $94 \%$ ee. The same reaction was also performed using diselenide $\mathbf{5}$ and proceeded faster (68 h vs 110 days) but considerably less enantioselective $(78 \%$ ee $)$. (Scheme 2). ${ }^{[9]}$

\section{Diselenides in Catalytic Oxidation}

More recently, we directed our interest to biomimetic catalytic applications of diselenides. In particular we exploited the ability of selenium in oxygen transfer reactions with a mechanism that mimics that of the enzyme glutathione peroxidase. Selenium-catalyzed epoxidations are well known processes ${ }^{[10]}$ and we recently demonstrated that in the presence of water a subsequent ring-opening reaction affords the formation of vicinal diols.[11] In this case the actual catalyst is a perseleninic acid generated by the oxidation of a diselenide in the presence of hydrogen peroxide. The epoxide intermediate is opened prevalently by an $\mathrm{S}_{\mathrm{N}} 2$-type reaction affording the anti-disubstituted derivatives stereoselectively. Using selenocystine (L$\mathrm{Sec}_{2}$ ) as a catalyst, the dimer of the natural amino acid, the reaction can be performed in water as medium allowing to recover and reuse the aqueous phase containing the catalyst for at least five cycles with only a moderate decrease in yield. ${ }^{[11 b]}$ The recyclability of the catalyst dissolved in the reaction medium in addition to the use of an eco-friendly oxidant like dilut-

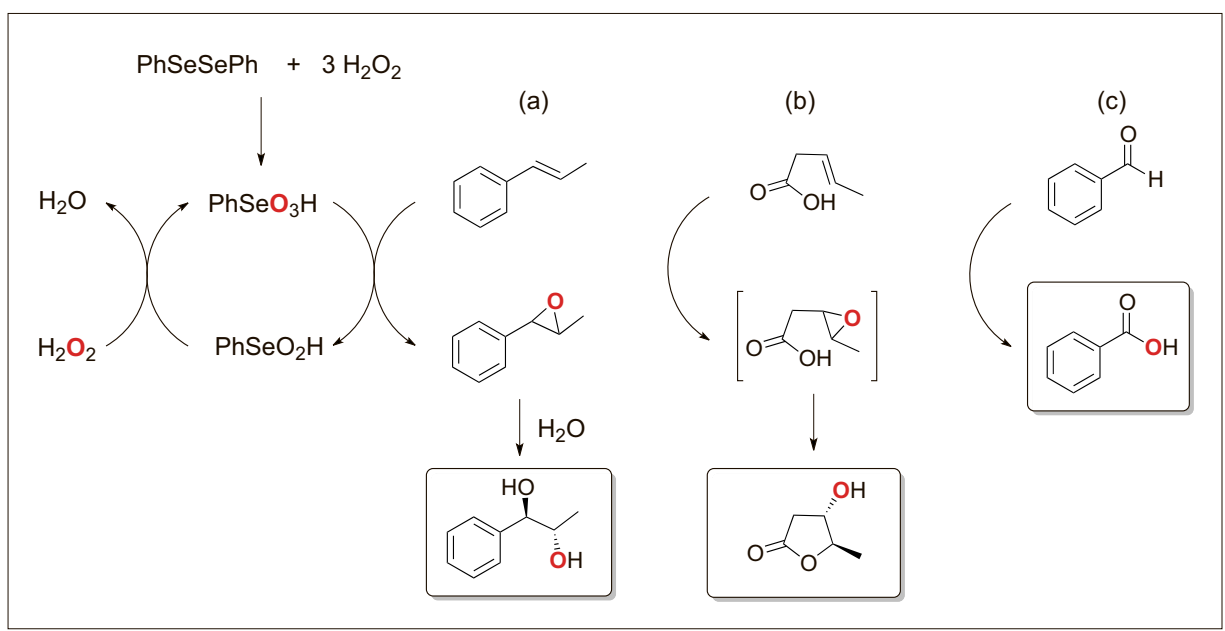

Scheme 3. Biomimetic catalysis: a) dihydroxylation, b) oxacyclofunctionalization, c) oxidation of aldehydes. internal nucleophile is an alcohol) in good yield in on water conditions (Scheme $3 \mathrm{~b}$ ). ${ }^{[12]}$ ed hydrogen peroxide positively affect the greenness of the reaction and prompted us to further investigate the applicability of these protocols in other oxidative conversions (Scheme 3).

When the oxygen transfer involves an olefin bearing, in a suitable position, a nucleophile able to promote the intramolecular ring opening of the epoxide, the reaction leads to the formation of functionalized heterocycles (hydroxy lactones if the nucleophile is a carboxylic acid or hydroxy tetrahydrofurans or tetrahydropyrans if the Also in these cases the reaction is highly stereoselective affording almost exclusively the trans-disubstituted compounds and, only in the presence of an aromatic substituent on the double bond, the ring opening via $\mathrm{S}_{\mathrm{N}} 1$ becomes competitive affording a syn/anti mixture in a ratio of 15:85. Very recently this reaction has been performed in continuous mode using a standard flow reactor and, to the best of our knowledge, this is the first example in which selenium catalysts have been successfully applied in flow conditions. ${ }^{[12 b]}$

Stoichiometric amounts of hydrogen peroxide in the presence of a catalytic amount of benzenselenenic acid promote the synthesis of variously functionalized 
carboxylic acids starting from the corresponding aldehydes in good to excellent yields (Scheme 3c). ${ }^{[13]}$ In most cases the reaction can be performed without the use of any organic solvents because the acids precipitate in water and can be recovered by filtration. In this way, starting from benzaldehyde, the catalyst and the water were recovered and reused five times performing the gram scale synthesis of benzoic acid in $87 \%$ yield and pharmaceuticalgrade purity. When an alcohol was used in the place of water the corresponding esters were obtained in very high yields suggesting the formation of a hemiacetal as intermediate in the reaction mechanism.

\section{Diselenides as Precursors of Nucleophilic Reagents}

Reductive cleavage of diselenides is a common procedure to form in situ nucleophilic selenium reagents that are versatile agents for the introduction of a selenium moiety into organic molecules. Several reducing agents, including $\mathrm{LiAlH}_{4}, \mathrm{NaBH}_{4}$, $\mathrm{Na} / \mathrm{NH}_{3}, \mathrm{Bu}_{3} \mathrm{SnH}$, have been reported under basic as well as neutral conditions for the preparation of selenates starting from diselenides. ${ }^{[14]}$ Our contribution in this field started ten years ago when we described a simple and efficient method to use zinc in a biphasic acidic system for the reduction of different diselenides and the reaction of the resulting selenate/selenol mixture with different electrophiles. ${ }^{[15]}$ This methodology has been successfully applied to a number of reactions: the ring opening of epoxides ${ }^{[15 a]}$ and aziridines, ${ }^{[16]}$ nucleophilic substitution of alkyl and acyl halides, ${ }^{[15 a, b]}$ hydroselenation ${ }^{[15 c]}$ and in the synthesis of peptides. ${ }^{[17]}$ An important extension of these studies resulted from the demonstration that, starting from the electrophilic $\mathrm{PhSeCl}$ or $\mathrm{PhSeBr}$ (that can be conveniently prepared from diphenyl diselenides), the treatment with elemental zinc produces the umpolung of the selenium atom affording the first class of benchstable selenates $\mathrm{PhSeZnCl}$ and $\mathrm{PhSeZnBr}$ recently named as Santi's reagents (SR). ${ }^{18]}$ An in-depth investigation of the reactivity of these reagents evidenced an unexpected rate acceleration under on water conditions for several classes of reactions (summarized in Scheme 4): i) catalytic aerobic oxidation of thiols into corresponding disulfides, ${ }^{[18 b]}$ ii) nucleophilic alkyl, aryl, vinyl and acyl substitution, ${ }^{[18 a, c, d]}$ iii) Michael-type addition reaction, ${ }^{[18 \mathrm{e}]}$ iv) ring opening of epoxides. ${ }^{[18 \mathrm{a}]}$ Braga and co-workers reported the use of the SR for the ring opening of aziridines ${ }^{[19]}$ but in this case it was necessary to use a ionic liquid as easily and efficiently recoverable medium.

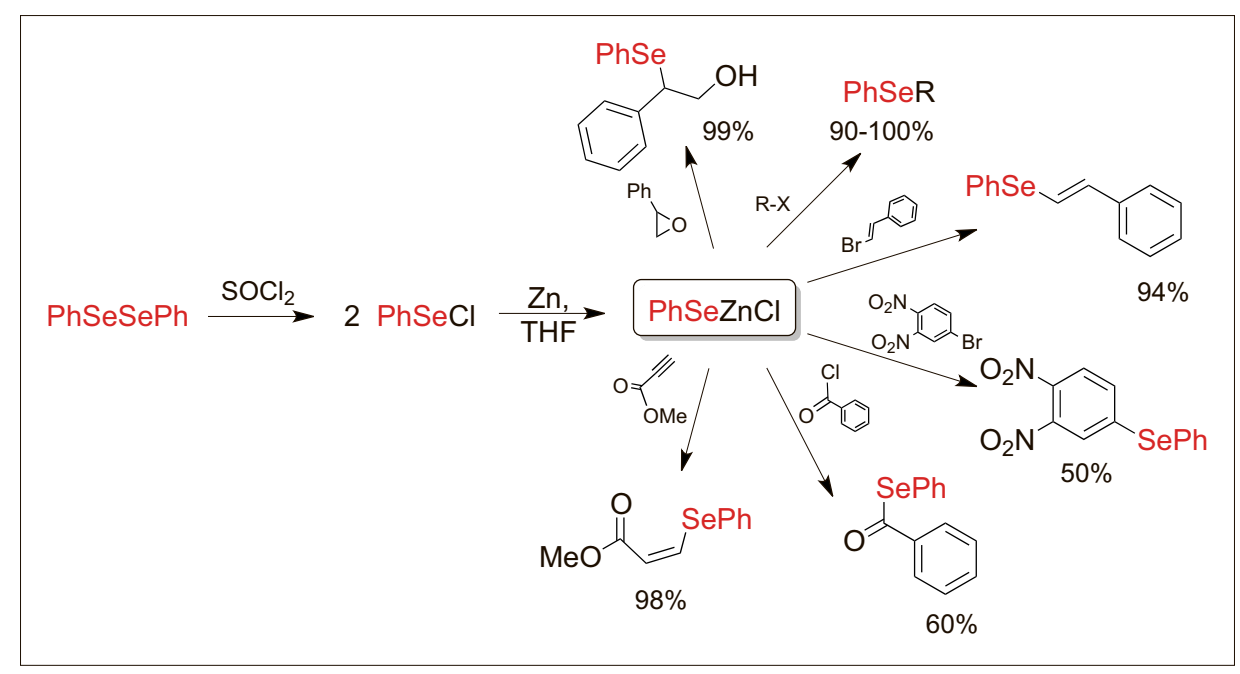

Scheme 4. Synthesis and on water reactivity of $\mathrm{PhSeZnCl}$.
Among the other applications reported in literature worth of mentioning is the use of $\mathrm{PhSeZnCl}$ in on water conditions for the ring opening of an epoxy keto ester as precursor of an allylic alcohol during the total synthesis of the biologically active metabolite idesolide. ${ }^{[20]}$

More recently the same reagent has been used for the post-synthetic modification of a poly(glycidyl methacrylate) polymer affording a series of derivatives with high refractive index that can be modulated by varying the selenium concentration. ${ }^{[21]}$ During the last year $\mathrm{PhSeZnCl}$ became commercially available, demonstrating the growing interest in the use of this reagent in organic synthesis mainly due to the possibility to enable selenenylation reactions in mild and easy conditions.

\section{Diselenides Endowed with Biological Activity}

Diaryl diselenides were reported to be mimics of glutathione peroxidase (GPx) and a number of different functionalities were investigated to better understand the lating the electrophilicity of the selenium atom and, as a direct consequence, its GPXlike activities. ${ }^{[1]}$

We investigated a series of diselenides (Fig. 2) functionalized with carboxyl groups in the ortho position with respect role of neighbouring heteroatoms in modu- to the selenium atom, obtaining structures (8 and 9) that showed a GPx-like activity largely higher than that observed for Ebselen (usually taken as a reference). ${ }^{[22]}$ Interestingly the same structures were also able to inhibit the formation and promote the dispersion of biofilms of bacteria and fungi envisioning the possibility of their application as good candidates of new therapeutic applications in acute wound infections as well as chronic skin diseases such as diabetic foot ulcers. ${ }^{[23]}$ Furthermore we also investigated a series of amido-decorated diaryl diselenides as inhibitors of the NucleoCapsid protein 7 (NCp7) that plays a crucial role in the replication of HIV virus. Diselenide $\mathbf{1 0}$ showed an appreciable selectivity index higher than the sulfur analogues. The antiviral activity of these diselenides is effectively due to the inhibition of the NCp7 plausibly by interacting with the zinc finger domain and promoting the extrusion of the metal. ${ }^{[24]}$

Finally, the diselenide $\mathbf{1 0}$ was selected to demonstrate, for the first time, the hormetic effect of a selenium-containing compound. Compound $\mathbf{1 0}$ is able to produce a mild oxidative stress stimulating the self-protection mechanism of the cell and activating the synthesis of proteins able to detoxify reactive oxygen species. The global result is an enhanced resistance of the cell to a stressogenic treatment with $\mathrm{H}_{2} \mathrm{O}_{2} \cdot{ }^{[25]}$

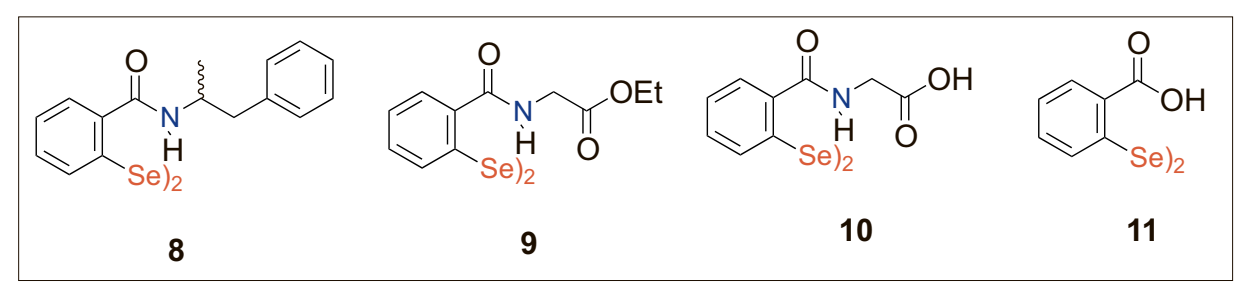

Fig. 2. Diselenides studied for their biological activity. 


\section{Acknowledgements}

The authors strongly thanks all the students that actively collaborated on the results summarized in this account, their names appear as co-authors in the list of references.

Received: July 27, 2017

[1] C. Santi, C. Tidei, C. Scalera, M. Piroddi, F. Galli, Curr. Chem. Biol. 2013, 7, 25.

[2] a) D.M. Browne, T. Wirth, Curr. Org. Chem. 2006, 10, 1893; b) 'Organoselenium Chemistry: Modern developments in organic synthesis', Ed. T. Wirth, Top. Curr. Chem. 2000, 208; c) 'Organoselenium Chemistry', Ed. T. Wirth, Wiley-VCH, 2011; d) F.V. Singh, T. Wirth, 'Stereoselective reactions of organoselenium reagents including catalysis', in Patai Series: 'Organic Selenium and Tellurium Compounds', Ed. Z. Rappoport, John Wiley \& Sons, 2012 Vol. 3, pp. 303-355; e) C. Santi, C. Tidei, 'Addition Reactions with Formation of CarbonSulfur and Carbon-Selenium Bonds', in 'Comprehensive Organic Synthesis', 2nd edition, Ed. G. A. Molander, P. Knochel, Elsevier, Oxford, 2014, Vol 7, pp. 605-637.

[3] C. Santi, T. Wirth Tetrahedron: Asymm. 1999, $10,1019$.

[4] C. Santi, G. Fragale, T. Wirth, Tetrahedron: Asymm. 1998, 9, 3625 .

[5] J. Ścianowski, Z, Rafiński, 'Electrophilic selenium reagents: addition reactions to double bonds and selenocyclizations', in 'Organoselenium Chemistry: Between Synthesis and Biochemistry', Ed. C. Santi, Bentham, 2014, pp 8-60 and references therein cited.

[6] M. Tiecco, L. Testaferri, C. Santi, C. Tomassini, F. Marini, L. Bagnoli, A. Temperini, Angew. Chem. 2003, 115, 3239.

[7] M. Tiecco, L. Testaferri, C. Santi, C. Tomassini, R. Bonini, F. Marini, L. Bagnoli, A. Temperini, Org. Lett. 2004, 6, 4751 .
[8] C. Tomassini, F. Di Sarra, B. Monti, L. Sancineto, L. Bagnoli, F. Marini, C. Santi, Arkivoc 2016, 2017, 303

[9] Electrophilic selenium: C Santi, S Santoro, in 'Organoselenium Chemistry: Synthesis and Reactions', Ed. T. Wirth, Wiley-VCH Verlag $\mathrm{GmbH} \&$ Co. KGaA, 2011, pp 1-51.

[10] a) B. Betzemeier, F. Lhermitte, P. Knochel, Synlett 1999, 489; b) G.-J. ten Brink, B. C. M. Fernandez, M. C. A. van Vliet, I. W. C. E. Arends, R. A. Sheldon, J. Chem. Soc. Perkin Trans. 1 2001, 224.

[11] a) S. Santoro, C. Santi, M. Sabatini, L. Testaferri, M. Tiecco, Adv. Synth. \& Cat. 2008, 350, 2881; b) C. Santi, R. Di Lorenzo, C. Tidei, L. Bagnoli, T. Wirth, Tetrahedron 2012, 68, 10530 .

[12] a) L. Sancineto, F. Mangiavacchi, C. Tidei, L. Bagnoli, F. Marini, A. Gioiello, J. Scianowski, C. Santi, Asian J. Org. Chem. 2017, DOI: 10.1002/ajoc.201700193; b) B. Cerra, F Mangiavacchi, C. Santi, A. M. Lozza, A. Gioiello, React. Chem. Eng. 2017, 2, 467.

[13] L. Sancineto, C. Tidei, L. Bagnoli, F. Marini, E. J. Lenardão, C. Santi, Molecules 2015, 20, 10496.

[14] a) K. K. Bashin, N. Singh, R. Kumar, D. G. Deepali, S. K. Metha, T. M. Klapoetke, M.-J. Crawford, J. Organomet. Chem. 2004, 689, 3327 ; b) M. Yoshimatsu, T. Sato, H. Shimizu, M. Hori, T. Kataoka, J. Org. Chem. 1994, 59, 1011; c) D. Crich, D. Grant, J. Org. Chem. 2005, 70, 2384; d) I. Andreadou, W. M. P. B. Menge, J. N. M. Commandeur, E. A. Worthington, N. P. E. Vermeulen, J. Med. Chem. 1996, 39, 2040; e) M. Sakakibara, K. Katsumata, Y. Watanabe, T. Toru, Y. Ueno, Synthesis 1992, 377; f) T. Wirth, 'Selenium', in 'Comprehensive Organometallic Chemistry' III, Eds. R. H. Crabtree, D. M. P. Mingos, Elsevier, Oxford, 2006, vol. 9, p. 457.

[15] a) C. Santi, S. Santoro, L. Testaferri, M. Tiecco, Synlett. 2008, 1471; b) G. Bellino, M. Scisciani, J. Pinto Vargas, L. Sancineto, L. Bagnoli, F. Marini, D. Seibert Lüdtke, E. Joao Lenardao,
C. Santi, J. Chemistry 2016, 2849140, doi:10.1155/2016/2849140; c) C. Tidei, L. Sancineto, L. Bagnoli,B. Battistelli, F. Marini, C. Santi, Eur. J. Org. Chem. 2014, 5968.

[16] A.L. Braga, R.S. Schwab, E.E. Alberto, S.M Salman, J. Vargas, J.B. Azeredo, Tetrahedron Lett. 2009, 50, 2309.

[17] a) S. Flemer, Prot. Pept. Lett. 2014, 21, 1257; b) S. Flemer, J. Pept. Sci. 2015, 21, 53.

[18] a) C. Santi, S. Santoro, B. Battistelli, L. Testaferri, M. Tiecco, Eur. J. Org. Chem. 2008, 32, 5387; b) C. Tidei, M. Piroddi, F. Galli, C. Santi, Tetrahedron Lett. 2012, 53, 232; c) S. Santoro, B. Battistelli, L. Testaferri, M. Tiecco, C. Santi, Eur. J. Org. Chem. 2009, 4921; d) C. Santi, B. Battistelli, L. Testaferri, M. Tiecco, Green Chem. 2012, 14, 1277; e) B. Battistelli, L. Testaferri, M. Tiecco, C. Santi Eur. J. Org. Chem. 2011, 1848.

[19] S. Narayanaperumal, E. E. Alberto, L. Gul, C. Y. Kawasoko, L. Dornelles, O. E. D. Rodrigues, A. L. Braga, Tetrahedron 2011, 67, 4723.

[20] M.-H. Jung, J.-M. Yoo, Y.-J. Kang, H. W. Lee, S. H. Kim, S. H. Sung, Y.-J. Lee, I. Choi, T.-J. Kim, Cells. Biol. Pharm. Bull. 2010, 33, 1063.

[21] H. Jiang, X. Pan, N. Li, Z. Zhang, J. Zhu, X. Zhu, React. Funct. Polymers 2017, 111, 1.

[22] V. Nascimento, N. L. Ferreira, F. S. Canto Romulo, K. L. Schott, E. P. Waczuk, L. Sancineto, C. Santi, J. B. T. Rocha, A. L. Braga, Eur. J. Med. Chem, 2014, 87, 131.

[23] L. Sancineto, M. Piccioni, S. De Marco, R. Pagiotti, V. Nascimento, A. L. Braga, C. Santi, D. Pietrella, BMC Microbiology 2016, 16, 220.

[24] L. Sancineto, A. Mariotti, L. Bagnoli, F. Marini, J. Desantis, N. Iraci, C. Santi, C. Pannecouque, O. Tabarrini, J. Med. Chem. 2015, 58, 9601.

[25] D. Bartolini, J. Commodi, M. Piroddi, L. Incipini, L. Sancineto, C. Santi, F. Galli Free Rad. Biol. \& Med. 2015, 88, 466. 